ИЗВЕСТИЯ АКАДЕМИИ НАУК ЭСТОНСКОИ ССР. ФИЗИКА * МАТЕМАТИКА PROCEEDINGS OF THE ACADEMY OF SCIENCES OF THE ESTONIAN SSR. PHYSICS * MATHEMATICS

$1988,37,3$

\title{
ПРОСТРАНСТВЕННО-ВРЕМЕННАЯ ГОЛОГРАФИЯ ВЕКТОРНОГО ПОЛЯ
}

В [ $\left.{ }^{1}\right]$ предложена и экспериментально реализована идея голографической записи и восстановления временной зависимости ультракороткого светового сигнала с помощью фотохромных сред, поддающихся фотовыжиганию узких спектральных провалов при низких температурах $\left[{ }^{2}\right]$. Это позволило обобщить обычную голографию с включением четвертого - временного измерения и тем самым развить пространственно-временную голографию изменяющихся во времени сцен $\left[{ }^{3-5}\right]$.

Однако нельзя ли пойти дальше и поставить задачу записи-воспроизведения поля следующим максималистским образом: запечатлеть пространственно-временную зависимость всех компонентов вектора электрического поля в среде в данной четырехмерной области, а при считывании восстановить пространственно-временное поведение не только амплитуды и фазы, но и поляризации вектора поля.

Методы получения голограмм, которые воспроизводят состояние поляризации в исходном стационарном поле, хорошо известны $\left[{ }^{6-9}\right]$. Для этих целей, например, весьма перспективно использование фотохромных сред, обладающих светоиндуцированным дихроизмом [ $\left.{ }^{10}\right]$.

Возможность голографирования временной зависимости амплитуды поля основывается на том, что поддающиеся фотовыжиганию примесные молекулы в среде имеют всевозможные резонансные частоты внутри неоднородной полосы поглощения и взамодействуют спектрально избирательно со светом, т. е. для каждого компонента Фурье (временной гармоники) светового сигнала в элементе среды существуют молекулы соответствующей резонансной частоты. Для того чтобы среда могла запечатлеть информацию об изменяющемся во времени состоянии поляризации сигнала, молекулы одной частоты должны дополнительно распределяться на две группы, избирательно взаимодействующие с двумя поляризационными компонентами временной гармоники. Последнее наблюдается, если молекулы имеют в среде разную (по крайней мере две ортогональные) ориентацию дипольного момента их электронного перехода и если эти ориентации стабильны (относительно теплового движения и т. п.). Наличие дихроизма и двулучепреломления в области провала, выжженного светом определенной поляризации, показывает, что фотоактивные примесные молекулы в стеклообраных матрицах распределяются по всевозможным направлениям дипольного момента и что наведенная светом анизотропия в распределении действительно сохраняется [ $\left.{ }^{11}\right]$. Эти эффекты используются в спектроскопических целях [ $\left.{ }^{11}\right]$, но они могут быть рассмотрены также как запечатление и восстановление одной спектрально-поляризационной компоненты световой волны.

Исходя из вышеизложенного, в $\left[{ }^{12}\right]$ был проведен теоретический анализ возможностей голографирования поля в полном смысле этого 
слова (учитывая и пространственно-временную зависимость поляризации вектора поля) на средах с фотовыжигаемыми примесными молекулами. В частности, было показано, что необращенная сигнальная волна с произвольной поляризацией восстанавливается без искажений при круговых поляризациях одинакового направления векторов поля опорного и считывающего импульсов, а обращенная волна - при противоположных направлениях поляризации этих векторов.

В настоящей работе изложена в приближении тонкой голограммы теория пространственно-временного голографирования векторного поля, основывающаяся на материалах изучения фотовыжигаемых примесных молекул.

\section{1. Диэлектрическая проницаемость анизотропной фотохромной среды пространственно-временной голограммы}

Пусть примесные молекулярные центры в фотохромной среде отличаются как по собственным частотам бесфононного перехода $\omega_{0}$, так и по ориентациям $\Omega$ дипольного момента того же перехода. Значения $\omega_{0}$ и $\Omega$ для каждой примесной молекулы фиксируются ее непосредственным матричным окружением *. Следовательно, индуцированный в молекуле дипольный момент, возникающий при взаимодействии со слабым внешним полем $\vec{E}(\vec{r}, t)$, имеет также направление $\Omega$. Его поведение удобно описывать с помощью уравнения классического гармонического осциллятора:

$$
\frac{\partial^{2}}{\partial t^{2}} R_{\Omega}(\vec{r}, t)+2 \Gamma \frac{\partial}{\partial t} R_{\Omega}(\vec{r}, t)+\omega_{0}^{2} R_{\Omega}(\vec{r}, t)=\frac{e}{m} \vec{E}(\vec{r}, t) \overrightarrow{e_{\Omega}},
$$

где $R_{\Omega}(\vec{r}, t)$ - амплитуда колебания осциллятора, расположенного в точке с радиус-вектором $\vec{i}$, и имеющего ориентацию дипольного момента в направлении единичного вектора $\overrightarrow{e_{\Omega}} ; \Gamma, \omega_{0}, e$ и $m-$ соответственно затухание, собственная частота колебаний, заряд и эффективная масса модельного осциллятора.

Оптический отклик среды описывается с помощью вектора макроскопической поляризации $\vec{P}(\vec{r}, t)$ :

$$
\vec{P}(\vec{r}, t)=e \int_{-\infty}^{\infty} \oint_{4 \pi} g_{\Omega}\left(\vec{r}, \omega_{0}\right) R_{\Omega}(\vec{r}, t) \vec{e}_{\Omega} d \Omega d \omega_{0},
$$

где $g_{\Omega}\left(\vec{r}, \omega_{0}\right)$ - совместная функция распределения примесных молекул по пространственным координатам, собственным частотам бесфононного перехода $\omega_{0}$ и ориентациям $\Omega$ дипольных моментов. Как видно, для получения $\vec{P}(\vec{r}, t)$ необходимо суммирование по молекулам с разными частотами перехода, а также усреднение по ориентациям дипольных моментов.

Электрическое поле в среде определяется уравнением Максвелла:

$$
\operatorname{rot} \operatorname{rot} \vec{E}(\vec{r}, t)+\frac{1}{c^{2}} \frac{\partial^{2}}{\partial t^{2}} \vec{E}(\vec{r}, t)+\frac{4 \pi}{c^{2}} \frac{\partial^{2}}{\partial t^{2}} \vec{P}(\vec{r}, t)=0
$$

где $c$ - скорость света в среде.

\footnotetext{
* Ориентация может кезначительно флуктуировать под действием теплового движения, она может также изменяться в самом процессе записи; для модели существенно лишь, чтобы переориентированные в процессе выжигания молекулы вышли из взаимодействия со светом в полосе частот сигнала.
} 
Д̆ля решения уравнений (1)-(3) воспользуемся обычным приемом преобразований Фурье:

$$
\begin{aligned}
& \vec{E}(\vec{r}, t)=\int_{-\infty}^{\infty} \vec{E}(\vec{r}, \omega) \exp [i \omega t] d \omega, \\
& R_{\Omega}(\vec{r}, t)=\int_{-\infty}^{\infty} R_{\Omega}(\vec{r}, \omega) \exp [i \omega t] d \omega .
\end{aligned}
$$

После соответствующих вычислений получим для фурье-амплитуды поля $\vec{E}(\vec{r}, \omega)$ уравнение в виде:

$$
\operatorname{rot} \operatorname{rot} \vec{E}(\vec{r}, \omega)-\frac{\omega^{2}}{c^{2}} \tilde{\varepsilon}(\vec{r}, \omega) \vec{E}(\vec{r}, \omega)=0,
$$

где $\tilde{\varepsilon}(\vec{r}, \omega)$ - тензор комплексной диэлектрической проницаемости среды

$$
\tilde{\varepsilon}(\vec{r}, \omega)=\tilde{I}+\frac{2 \pi e^{2}}{m \omega} \int_{-\infty}^{\infty} \oint_{4 \pi} \frac{g_{\Omega}\left(\vec{r}, \omega_{0}\right)\left(\overrightarrow{e_{\Omega}} \times \overrightarrow{e_{\Omega}}\right)}{\omega_{0}-\omega+i \Gamma} d \Omega d \omega_{0},
$$

а $I-$ единичный тензор. Через $\vec{e}_{\Omega} \times \vec{e}_{\Omega}$ здесь обозначено тензорное произведение векторов. При выводе (5)-(6) учтено условие резонанса $\omega_{0} \approx_{\omega}$, т. е. заменено $\omega_{0}^{2}-\omega^{2} \sim 2 \omega\left(\omega_{0}-\omega\right)$.

Полученное выражение (6) для комплексной диэлектрической проницаемости среды обобщает соответствующее выражение в $\left[{ }^{3}\right]$. Поскольку у нас фотохромная среда может быть и анизотропной, то вместо скалярной величины, рассматриваемой в $\left[{ }^{3}\right]$, диэлектрическая проницаемость среды теперь является симметричным тензором второго ранга.

В дальнейшем необходимо определить явный вид функции распределения $g_{\Omega}\left(\vec{r}, \omega_{0}\right)$. Последняя зависит от структуры голограммы, записанной в среде посредством фотовыжигания провалов, причем степень модуляции $g_{\Omega}\left(\vec{r}, \omega_{0}\right)$ в первом приближении пропорциональна спектральной интенсивности $|\vec{A}(\vec{r}, \omega)|^{2}$ применяемого для записи света $\left[{ }^{3,13}\right]$. Однако теперь необходимо дополнительно учитывать зависимость вероятности перехода молекул на возбужденный уровень (с последующим их фотовыжиганием) от ориентации $\Omega$ его дипольного момента относительно вектора спектральной амплитуды $\vec{A}(\vec{r}, \omega)$ электрического поля возбуждающего света. Поскольку вероятность перехода пропорциональна квадрату косинуса угла между названными векторами, распределение молекул после записи голограммы выражается:

$$
g_{\Omega}\left(\vec{r}, \omega_{0}\right)=g_{0}\left(1-x t_{E}\left|\vec{A}\left(\vec{r}, \omega_{0}\right) \vec{e}_{\Omega}\right|^{2}\right),
$$

где $g_{0}$ - распределение примесных молекул до экспозиции, предполагаемое постоянной, $x-$ коэффициент выжигания, $t_{E}-$ время экспозиции. В (7) предположено выполнение условия малой глубины выжигания

$$
x t_{E}|\vec{A}(\vec{r}, \omega)|^{2} \ll 1 .
$$

Если с учетом (7) провести в (6) процедуру усреднения по углам ориентации $\Omega$, (подробнее см., напр., $\left[{ }^{14}\right]$, а также $\left[{ }^{15}\right]$, то находим: 


$$
\oint_{4 \pi}\left(\vec{e}_{\Omega} \times e_{\Omega}\right) d \Omega=\frac{4 \pi}{3} T
$$

и

$$
\begin{gathered}
\oint_{4 \pi}\left|\vec{A}(\vec{r}, \omega) \overrightarrow{e_{\Omega}}\right|^{2}\left(\overrightarrow{e_{\Omega}} \times \overrightarrow{e_{\Omega}}\right) d \Omega=\frac{4 \pi}{15}\left[|\vec{A}(\vec{r}, \omega)|^{2} \widetilde{I}+\right. \\
\left.+\vec{A}(\vec{r}, \omega) \times \overrightarrow{A^{*}}(\vec{r}, \omega)+\vec{A}^{*}(\vec{r}, \omega) \times \vec{A}(\vec{r}, \omega)\right] .
\end{gathered}
$$

Здесь звездочка означает комплексно сопряженные величины. Далее, после использования символического равенства

$$
\lim _{\Gamma \rightarrow 0} \frac{1}{\omega_{0}-\omega+i \Gamma}=-i \pi \delta\left(\omega_{0}-\omega\right)+P\left(\frac{1}{\omega_{0}-\omega}\right)
$$

и проведения в (6) интегрирования по $\omega_{0}$ окончательно находим для тензора комплексной диэлектрической проницаемости среды

$$
\begin{gathered}
\tilde{\varepsilon}(\vec{r}, \omega)=I+\frac{8 \pi^{3} i e^{2} g_{0}}{3 m \omega}\left\{-I+\frac{(1+i \hat{H}) x t_{E}}{5} \times\right. \\
\left.\left.\times\left[|\vec{A}(\vec{r}, \omega)|^{2} \tau+\vec{A}(\vec{r}, \omega) \times \vec{A} * \vec{r}, \omega\right)+\vec{A}^{*}(\vec{r}, \omega) \times \vec{A}(\vec{r}, \omega)\right]\right\},
\end{gathered}
$$

где $\hat{H} f(\omega)$ - гилберт-образ функции $f(\omega),|\vec{A}(\vec{r}, \omega)|^{2}-$ квадрат абсолютного значения вектора $\vec{A}(\vec{r}, \omega)$ :

$$
|\vec{A}(\vec{r}, \omega)|^{2}=\left|A_{x}(\vec{r}, \omega)\right|^{2}+\left|A_{y}(\vec{r}, \omega)\right|^{2}+\left|A_{z}(\vec{r}, \omega)\right|^{2},
$$

$\vec{A}(\vec{r}, \omega) \times \vec{A}^{*}(\vec{r}, \omega)$ и $\overrightarrow{A^{*}}(\vec{r}, \omega) \times \vec{A}(\vec{r}, \omega)$ - транспонированные относительно друг-друга тензоры, полученные при прямом умножении векторов, например:

$$
\vec{A}(\vec{r}, \omega) \times \vec{A}^{*}(\vec{r}, \omega)=\left|\begin{array}{lll}
\left|A_{x}(\vec{r}, \omega)^{2}\right| & A_{x}(\vec{r}, \omega) A_{y}^{*}(\vec{r}, \omega) & A_{x}(\vec{r}, \omega) A_{z}^{*}(\vec{r}, \omega) \\
A_{y}(\vec{r}, \omega) A_{x}^{*}(\vec{r}, \omega) & \left|A_{y}(\vec{r}, \omega)\right|^{2} & A_{y}(\vec{r}, \omega) A_{z}^{*}(\vec{r}, \omega) \\
A_{z}(\vec{r}, \omega) A_{x}^{*}(\vec{r}, \omega) & A_{z}(\vec{r}, \omega) A_{y}^{*}(\vec{r}, \omega) & \left|A_{z}(\vec{r}, \omega)\right|^{2}
\end{array}\right|
$$

\section{2. Решение волнового уравнения для пластинки голограммы}

Приступим теперь к решению волнового уравнения (5). Коорди: натные оси выберем так, чтобы фотохромная среда в виде пластинки находилась между плоскостями $z=0$ и $z=d$ (см. рисунок). Пластинку-голограмму считаем тонкой. Далее, поскольку анизотропность и пространственная неоднородность фотохромной среды считаются незначительными в смысле соотношения $\left(7^{\prime}\right)$, падающее световое поле внутри пластинки целесообразно представить в виде:

$$
\vec{E}(\vec{r}, \omega)=\vec{E}^{M}(\vec{r}, \omega) \exp [-i \vec{k} \vec{r}],
$$


где $\vec{E}^{M}(\vec{r}, \omega)$ - медленно изменяющаяся амплитуда: $\quad \vec{E}^{M}(\vec{r}, \omega)=$ $=\left(E_{x}^{M}(\vec{r}, \omega), E_{y}^{M}(\vec{r}, \omega), E_{z}^{M}(\vec{r}, \omega)\right), \exp [-i \vec{k} \vec{r}]$ - быстро осциллирующая фаза, $\vec{k}$ - среднее значение волнового вектора света в среде:



Запись пространственно-временной голограммы векторного поля. Голограмма выжигается посредством интерференции сигнального импульса $\vec{S}(\vec{r}, t)$ в любой форме и $\delta$-образного опорного импульса $R(\vec{r}, t)$ с плоским волновым фронтом, имеющим круговую поляризацию в среде пластинки голограммы. $d$ - толщина пластинки, $t_{R}$ и $\Theta-$ соответственно, задержка и угол отклонения опорного импульса по отношению к сигнальному.

$|\vec{k}|=\frac{\omega}{c}$. Как известно $\left[{ }^{14}\right]$, в общем случае при распространении светового поля в неоднородной и анизотропной среде как направление, так и абсолютное значение его волнового вектора $\vec{k}$ не являются постоянными. В нашем случае соответствующие изменения малы по сравнению со средним значением волнового вектора. Поэтому они учитываются в медленном множителе $\vec{E} \vec{E}^{M}(\vec{r}, \omega)$. Согласно $(5)$, нам необходимо дважды воздействовать оператором rot на выражение (13). Для упрощения вычислений предлагаем считывание пластинки проводить со светом, распространяющимся под малым углом $\Theta \leqslant 2^{\circ}-3^{\circ}$ относительно оси $z$. В таком случае вектор электрического поля падающего света при прохождении пластинки всегда практически поляризованный в $(x, y)$-плоскости. Следовательно, для компонентов амплитуды вектора электрического поля можно считать

$$
\left|E_{z}^{M}(\vec{r}, \omega)\right| \ll\left|E_{x}^{M}(\vec{r}, \omega)\right|,\left|E_{y}^{M}(\vec{r}, \omega)\right| .
$$

При этом компоненты волнового вектора $\vec{k}=\left(k_{x_{1}}, k_{y_{1}}, k_{z}\right)$, наоборот, удовлетворяют условиям

$$
k_{y}, k_{x} \ll k_{z} .
$$

Воспользуемся также приближением медленноизменяющихся амплитуд, т. е. предположим выполненными условия

$$
\frac{\partial^{2}}{\partial x_{L} \partial x_{k}} E_{i}^{M}(\vec{r}, \omega) \ll|\vec{k}|^{2},
$$

где $i=x, y, z ; x_{L}, x_{k}=x, y, z$.

2 ENSV TA Toimetised. F* M 31988 
$\widehat{\mathrm{B}}$ сделанных приближениях находим

$\operatorname{rot} \operatorname{rot} \vec{E}(\vec{r}, \omega) \approx\left[2 i k_{z} \frac{\partial}{\partial z} \vec{E}^{M}(\vec{r}, \omega)+|\vec{k}|^{2} \vec{E}^{M}(\vec{r}, \omega)\right] \exp [-i \vec{k} \vec{r}]$,

где $k_{z}-z-$ компонента волнового вектора $\vec{k}$; в дальнейшем считаем $k_{z} \approx|\vec{k}|=\frac{\omega}{c}$. Подставляя (11), (13) и (16) в (5), получаем

$$
\begin{aligned}
& \frac{\partial}{\partial z} \vec{E}^{M}(\vec{r}, \omega)+\frac{4 \pi^{3} e^{2} g_{0}}{3 m c}\left\{I-\frac{(1+i \hat{H}) x t_{E}}{5}\left[|\vec{A}(\vec{r}, \omega)|^{2} \cdot T+\right.\right. \\
& \left.\left.+\vec{A}(\vec{r}, \omega) \times \vec{A}^{*}(\vec{r}, \omega)+\vec{A}^{*}(\vec{r}, \omega) \times \vec{A}(\vec{r}, \omega)\right]\right\} \vec{E}^{M}(\vec{r}, \omega)=0 .
\end{aligned}
$$

Поскольку пластинка, предположительно, оптически тонкая, допустима замена производной в (17) на полное изменение поля при прохождении пластинки

$$
\frac{\partial}{\partial z} \vec{E}^{M}(\vec{r}, \omega) \rightarrow \frac{\vec{E}_{d}^{M}(\vec{r}, \omega)-\vec{E}_{0}^{M}(\vec{r}, \omega)}{d}
$$

где $d-$ толщина пластинки, $\vec{E}_{0}^{M}(\vec{r}, \omega)$ и $\vec{E}_{d}^{M}(\vec{r}, \omega)$ - амплитуды электрического вектора светового поля соответственно до (в плоскости $z=0$ ) и после прохождения (в плоскости $z=d$ ) пластинки. Подставляя (18) в (17), умножая полученное выражение на фазовый множитель $\exp [-i \vec{k} \vec{r}]$ и вычисляя затем обратное преобразование Фурье, находим выражение для определения полной пространственно-временной зависимости светового поля, восстановленного с голограммы. При вычислениях учитываем, что обратный фурье-образ выражения $(1+i \hat{H}) f(\omega)$ есть $2 \Upsilon(t) f(t)$, где $\Upsilon(t)$ - единичная ступенька Хэвисайда, а также то, что обратный фурье-образ произведения двух функций выражается в виде свертки, а обратный фурье-образ произведения функций на комплексно-сопряженную функцию - в виде кросс-корреляции исходных функций. В итоге для выходного поля находим:

$$
\begin{aligned}
& \vec{E}_{d}(\vec{r}, t)=\left(1-N_{1}\right) \vec{E}_{0}(\vec{r}, t)+N_{2} \int_{\infty}^{\infty} \Upsilon\left(t_{1}\right) \int_{\infty}^{\infty}\left[\overrightarrow{A^{*}}\left(\vec{r}, t_{2}\right) \vec{A}\left(\vec{r}, t_{1}+t_{2}\right) \cdot \widetilde{I}+\right. \\
& \left.+\vec{A}\left(\vec{r}, t_{2}+t_{1}\right) \times \vec{A}^{*}\left(\vec{r}, t_{2}\right)+\vec{A}^{*}\left(\vec{r}, t_{2}\right) \times \vec{A}\left(\vec{r}, t_{2}+t_{1}\right)\right] \vec{E}_{0}\left(\vec{r}, t-t_{1}\right) d t_{2} d t_{1},
\end{aligned}
$$

где $\vec{E}_{0}(\vec{r}, t)$ и $\vec{E}_{d}(\vec{r}, t)$ - электрические векторы светового поля соответственно до и после прохождения пластинки;

$N_{1}=\frac{4 \pi^{3} e^{2} d g_{0}}{3 m c}, N_{2}=\frac{2 x t_{E} N_{1}}{5}$.

\section{3. Запись и восстановление поляризационной зависимости светового сигнала}

Приступим теперь к исследованию вопроса о возможности голографической записи и восстановления поляризационной структуры светового сигнала. При этом учтем, что световое поле $A(\vec{r}, \vec{t})$ в (19) состоит из опорного и сигнального импульсов

$$
\vec{A}(\vec{r}, t)=\vec{R}(\vec{r}, t)+\vec{S}(\vec{r}, t)
$$


Сигнальный импульс $\vec{S}(\vec{r}, t)$ пусть распространяется в направлений оси $z$ и имеет сложную пространственную структуру (см. рисунок):

$$
\begin{gathered}
\vec{S}(\vec{r}, t)=\left[S_{x}\left(x, y, t-\frac{z}{c}\right) \vec{e}_{x}+S_{y}\left(x, y, t-\frac{z}{c}\right) \vec{e}_{y}\right] \times \\
\times \exp \left[i \omega_{k}\left(t-\frac{z}{c}\right)\right]
\end{gathered}
$$

где $\overrightarrow{e_{x}}$ и $\overrightarrow{e_{y}} \quad$ - единичные векторы по направлениям осей $x$ и $y$ соответственно, $\omega_{k}$ - несущая частота импульса. Мгновенная поляризация поля (21) в каждой точке пространства определяется отношением комплексных амплитуд $S_{x}\left(x, y, t-\frac{z}{c}\right)$ и $S_{y}\left(x, y, t-\frac{z}{c}\right)$, причем запись в виде $(21)$ в общем случае учитывает возможность пространственно-временной модуляции всех характеристик (амплитуды, фазы, частоты и поляризации) данного светового сигнала.

Опорный импульс $\vec{R}(\vec{r}, t)$ выберем в виде импульса плоской волны, распространяющейся под малым углом $\theta$ по отношению к оси $z$ в $(x, z)$-плоскости:

$$
\begin{aligned}
\vec{R}(\vec{r}, t)= & {\left[R_{x} \vec{e}_{x}+R_{y} \exp \left[i \Phi_{R}\right] \overrightarrow{e_{y}}\right] \delta\left(t-\frac{\theta x+z}{c}-t_{R}\right) \times } \\
& \times \exp \left[i \omega_{k}\left(t-\frac{\theta x+z}{c}-t_{R}\right)\right],
\end{aligned}
$$

где $t_{R}$ - задержка опорного импульса относительно переднего фронта сигнального импульса, $R_{x}$ и $R_{y}$ - амплитуды компонентов электрического поля. Под огибающей $\delta$-функцией подразумевается исчезающе малая длительность опорного импульса по сравнению, с сигнальным (подробнее см. $\left.\left[{ }^{3,4}\right]\right)$. Фазой $\Phi_{R}$ определяется поляризация опорного импульса: (I) в общем случае: $R_{x} \neq R_{y} \neq 0,-\pi<\Phi_{R}<\pi$ она эллиптическая; (II) если $R_{x}=R_{y}$ и $\Phi_{R}=-\frac{\pi}{2}$ или $\Phi_{R}=\frac{\pi}{2}-$ поляризация круговая, соответственно по и против часовой стрелки (если смотреть в направлении распространения луча); (III) если $\Phi_{R}=0, \pi$, или $R_{y}=0$, или $R_{x}=0$ - поляризация линейная в $(x, y)$-плоскости. Ввиду малости угла $\theta$ при записи (22), согласно (14), не учитывается компонент вектора поля вдоль направления оси $z$. Считывающий импульс $\vec{E}_{0}(\vec{r}, t)$ следует выбирать аналогичным опорному:

$$
\begin{aligned}
\vec{E}_{0}(\vec{r}, t)= & {\left[E_{0 x} \vec{e}_{x}+E_{0 y} \exp \left[i \Phi_{L}\right] \vec{e}_{y}\right] \delta\left(t-\frac{\theta x+z}{c}\right) \times } \\
& \times \exp \left[i \omega_{k}\left(t-\frac{\theta x+z}{c}\right)\right],
\end{aligned}
$$

где $\Phi_{L}$ определяет поляризацию.

Поскольку согласно (21) и $(22)$ в записываемом поле $\vec{A}(\vec{i}, t)$ отсутствует компонент вдоль оси $z$, формула (19) значительно упрощается, так как в ней сохраняются всего лишь четыре отличные от нуля корреляционные функции. Иепользуя (21) и (22), находим:

1. $A_{x}^{*} * A_{x}=\left[S_{x}^{*}(x, y, t) * S_{x}(x, y, t)+R_{x}^{2} \delta(t)\right] \exp \left[i \omega_{k} t\right]+$ $+R_{x} S_{x}^{*}\left(x, y, \tau_{1}\right) \exp \left[-i \omega_{k} \tau_{1}\right]+R_{x} S_{x}\left(x, y, \tau_{2}\right) \exp \left[i \omega_{k} \tau_{2}\right] ;$ 
2. $A_{y}^{*} * A_{y}=\left[S_{y}^{*}(x, y, t) * S_{y}(x, y, t)+R_{y}^{2} \delta(t)\right] \exp \left[i \omega_{k} t\right]+$ $+R_{y} S_{y}^{*}\left(x, y, \tau_{1}\right) \exp \left[-i \omega_{k} \tau_{1}+i \Phi_{R}\right]+R_{y} S_{y}\left(x, y, \tau_{2}\right) \exp \left[i \omega_{k} \tau_{2}-i \Phi_{R}\right]$;

3. $A_{x}^{*} * A_{y}=\left[S_{x}^{*}(x, y, t) * S_{y}(x, y, t)+R_{x} R_{y} \delta(t) \exp \left[i \Phi_{R}\right]\right] \times$ $\times \exp \left[i \omega_{k} t\right]+R_{y} S_{x}^{*}\left(x, y, \tau_{1}\right) \exp \left[-i \omega_{k} \tau_{1}+i \Phi_{R}\right]+$ $+R_{x} S_{y}\left(x, y, \tau_{2}\right) \exp \left[i \omega_{k} \tau_{2}\right]$

4. $A_{y}^{*} * A_{x}=\left[S_{y}^{*}(x, y, t) * S_{x}(x, y, t)+R_{x} R_{y} \delta(t) \exp \left(-i \Phi_{R}\right)\right] \exp \left(i \omega_{k} t\right)+$ $+R_{x} S_{y}^{*}\left(x, y, \tau_{1}\right) \exp \left(-i \omega_{k} \tau_{1}\right)+R_{y} S_{x}\left(x, y, \tau_{2}\right) \exp \left(i \omega_{k} \tau_{2}-i \Phi_{R}\right)$,

где $A_{x}=A_{x}(\vec{r}, t), \quad A_{y}=A_{y}(\vec{r}, t), \quad \tau_{1}=-t+\frac{\theta x}{c}+t_{R}, \quad \tau_{2}=t+\frac{\theta x}{c}+t_{R}$ и * обозначает операцию кросс-корреляции:

$$
f_{1}^{*}(t) * f_{2}(t)=\int_{-\infty}^{\infty} f_{1}(\tau) f_{2}(t+\tau) d \tau .
$$

Подставляя найденные выражения в (19) и вычисляя свертки, получим выражение для определения суммарного светового поля за пластинкой. Как правило, при голографическом восстановлении изображения основной интерес представляют только члены, описывающие световые пучки, отклоненные от первоначального направления распространения при прохождении голограммы. В формулах (24) данные члены узнаваемы по аргументам $\tau_{1}$ и $\tau_{2}$. А именно, члены, содержащие среди аргументов переменную $\tau_{1}$, описывают пучки, дифрагированные под углом $2 \theta$ (т. е. соответствуют действительному (псевдоскопическому) изображению источника записанного сигнала), а члены, содержащие переменную $\tau_{2}$ - пучки, дифрагированные в направлении оси $z$ (в направлении сигнального импульса). Отмеченные последними члены соответствуют мнимому изображению источника сигнала.

Рассмотрим сперва прямое восстановление сигнала, т. е. восстановление мнимого изображения. Учитывая в (24) только соответствующие члены и вычисляя согласно (19) их свертки со считывающим импульсом (23), находим:

$$
\begin{aligned}
\vec{E}_{\mathrm{MH} .}(\vec{r}, t) & =N_{2} \Upsilon\left(t-\frac{\theta x+z}{c}\right) \exp \left[i \omega_{k} \tau\right]\left\{\left[3 R_{x} E_{0 x} S_{x}+R_{y} E_{0 x} S_{y} \exp \left[-i \Phi_{R}\right]+\right.\right. \\
& \left.+R_{x} E_{0 y} S_{y} \exp \left[i \Phi_{L}\right]+R_{y} E_{0 y} S_{x} \exp \left[i \Phi_{L}-i \Phi_{R}\right]\right] \vec{e}_{x}+ \\
& +\left[3 R_{y} E_{0 y} S_{y} \exp \left[i \Phi_{L}-i \Phi_{R}\right]+R_{x} E_{0 y} S_{x} \exp \left[i \Phi_{L}\right]+\right. \\
& \left.\left.+R_{x} E_{0 x} S_{y}+R_{y} E_{0 x} S_{x} \exp \left[-i \Phi_{R}\right]\right] \vec{e}_{y}\right\}
\end{aligned}
$$

где $S_{y}=S_{y}(x, y, \tau), S_{x}=S_{x}(x, y, \tau)$ и $\tau=t-\frac{z}{c}+t_{R}$.

Первоочередной интерес представляют условия, при которых записанный сигнал (21) восстанавливается полностью и без искажений. Нетрудно убедиться, что при выполнении условий:

1. $E_{0 y}=E_{0 x}, R_{y}=R_{x}$,

2. $\left\{\begin{array}{l}\Phi_{L}+\Phi_{R}= \pm \pi \\ \Phi_{L}-\Phi_{R}=0\end{array} \Rightarrow \Phi_{R}= \pm \frac{\pi}{2},-\Phi_{L}= \pm \frac{\pi}{2}\right.$ 
возможно правильное воспроизведение поляризации исходного сигнала. Действительно, в таком случае

$$
\begin{gathered}
\vec{E}_{\mathrm{MH},}(\vec{r}, t)=4 N_{2} \Upsilon\left(t-\frac{\theta x+z}{c}\right) \cdot R_{x} E_{0 x}\left[S_{x}\left(x, y, t-\frac{z}{c}+t_{R}\right) \vec{e}_{x}+\right. \\
\left.+S_{y}\left(x, y, t-\frac{z}{c}+t_{R}\right) \vec{e}_{y}\right] \exp \left[i_{\omega_{k}}\left(t-\frac{z}{c}+t_{R}\right)\right] .
\end{gathered}
$$

Қак видно, полученное выражение качественно отличается от выражения (21) лишь наличием функции $\Upsilon(t)$ во временной зависимости. Ее роль - обеспечение выполнения условия причинности при считывании голограммы $\left[{ }^{3,4}\right]$. Если $t_{R}<0$, т. е. при записи опорный импульс подавался на пластинку до сигнального, то функция $\Upsilon(t)$ в $(28)$ оказывается лишней. В результате происходит полное восстановление записанного поля, т. е. восстановление пространственных, временных и поляризационных характеристик сигнала.

Перейдем теперь к рассмотрению восстановления действительного изображения. Учитывая в (24) и (19) только члены, содержащие переменную $\tau_{1}$, находим после вычисления свертки:

$$
\begin{aligned}
& \vec{E}_{\text {дейст. }}(\vec{r}, t)=N_{2} \Upsilon\left(t-\frac{\theta x+z}{c}\right) \exp \left[-i \omega_{k} \tau\right]\left\{\left[3 R_{x} S_{x}^{*} E_{0 x}+\right.\right. \\
& \left.+R_{y} E_{0 x} S_{y}^{*} \exp \left[i \Phi_{R}\right]+R_{y} E_{0 y} S_{x}^{*} \exp \left[i \Phi_{L}+i \Phi_{R}\right]+R_{x} E_{0 y} S_{y}^{*} \exp \left[i \Phi_{L}\right]\right] \vec{e}_{x}+ \\
& +\left[3 R_{y} E_{0 y} S_{y}^{*} \exp \left[i \Phi_{L}+i \Phi_{R}\right]+R_{x} E_{0 y} S_{x}^{*} \exp \left[i \Phi_{L}\right]+\right. \\
& \left.\left.+R_{y} E_{0 x} S_{x}^{*} \exp \left[i \Phi_{R}\right]+R_{x} E_{0 x} S_{y}^{*}\right] \vec{e}_{y}\right\}
\end{aligned}
$$

где $S_{x}^{*}=S_{x}^{*}(x, y, \tau), S_{y}^{*}=S_{y}^{*}(x, y, \tau)$ и $\tau=-t+\frac{2 \theta x+z}{c}+t_{R}$.

Условия правильного восстановления поляризации теперь следующие:

1. $E_{0 y}=E_{0 x}, R_{x}=R_{y}$,

$$
\left\{\begin{array}{l}
\Phi_{L}-\Phi_{R}= \pm \pi \\
\Phi_{L}+\Phi_{R}=0
\end{array} \Rightarrow \Phi_{L}= \pm \frac{\pi}{2}, \quad \Phi_{R}=\mp \frac{\pi}{2} .\right.
$$

При этом за пластинкой восстанавливается поле

$$
\begin{aligned}
& \vec{E}_{\text {дейст. }}(\vec{r}, t)=2 N_{2} \Upsilon\left(t-\frac{\theta x+z}{c}\right) R_{x} E_{0 x} \exp \left[-i \omega_{k}(-t+\right. \\
& \left.\left.+\frac{2 \theta x+z}{c}+t_{R}\right)\right] \cdot\left[S_{x}^{*}\left(x, y,-t+\frac{2 \theta x+z}{c}+t_{R}\right) \vec{e}_{x}\right. \\
& \left.+S_{y}^{*}\left(x, y,-t+\frac{2 \theta x+z}{c}+t_{R}\right) \vec{e}_{y}\right]
\end{aligned}
$$

Световой сигнал (34) возникает только при выполнении условия $t_{R}>0$ (подробнее см. $\left.\left[{ }^{3,4}\right]\right)$, т. е. при записи опорный импульс должен подаваться на пластинку после сигнального. Как видно, под углом $2 \Theta$ восстанавливается сигнал (21) в сопряженной формс с обращенным ходом времени, а также с обращенным направлением хода поляризации, 


\section{4. Заключение}

Приходим к следующим выводам в отношении возможностей голографирования векторного поля с помощью высокоселективно фотохромных сред. Полное восстановление светового сигнала, включающее восстановление пространственно-временной зависимости как амплитуды вектора поля, так и его направления (поляризации), имеет место:

1. В виде прямой (необращенной) волны, если во время записи опорный импульс подавался на пластинку до сигнального и если при этом опорный и считывающий импульсы имеют круговую поляризацию одинакового направления.

2. В виде сопряженной (с обращенным ходом времени) волны, если во время записи опорный импульс подавался на пластинку после сигнального и если при этом опорный и считывающий импульсы имели круговую поляризацию взаимно противоположного направления.

3. При прочнх поляризациях опорного и считывающего импульсов восстановление происходит перепутыванием поляризационных составляющих сигнального поля.

В итоге, благодаря средам с селективно фотовыжигаемыми примесными молекулами открывается путь к реализации такой голографии, которая в точности соответствует буквальному смыслу этого термина - «полная запись». Образно говоря, светочувствительность этих сред является пятиразмерной, так как светочувствительные центры распределены в них как по трем пространственным измерениям, так и по резонансным частотам и ориентациям дипольного момента электронного перехода. Объемное распределение центров позволяет записывать пространственную зависимость поля, частотное распределение центров - его модуляцию во времени, а ориентационное распределение - направление вектора поля. Существенно новым моментом для голографий является то, что совместное частотно-ориентационное распределение центров позволяет записывать и воспроизводить любую временную зависимость состояния поляризации поля, например, поворот во времени плоскости линейной поляризации.

Первые экспериментальные результаты, подтверждающие выводы изложенной теории, получены в $\left[{ }^{16,17}\right]$.

Авторы благодарны К. К. Ребане за полезные советы и Р. Каарли за содействие в завершении работы и обсуждении результатов.

Л ИТ Р Р А У Р А

1. Ребане А. К., Каарли Р. К., Саари П. М. Письма в ЖЭТФ, 38, вып. 7, 320-323 (1983).

2. Гороховский А. А., Каарли Р. К., Ребане Л. А. Письма в ЖЭТФ, 20, вып. 7, $474-479$ (1974)

3. Саари П., Ребане А. Изв. АН ЭССР. Фнз. Матем., 33, № 3, 322-333 (1984).

4. Саари П. М., Каарли Р. К., Ребане А. К. Квант. электроника, 12, № 4, 672-682 (1985)

5. Saari, P., Kaarli, R., Rebane, A. J. Opt. Soc. Amer. B, 3, № 4, 527-533 (1986)

6. Lohman, A. Appl. Optics, 4, № 10, 1667-1674 (1965).

7. Дерючин И. А., Кураиов В. Н., Поданчук Д. В., Хороиков Ю. В. УФН, 108, вып. 4, 733-747 (1972).

8. Денисюк Ю. Н. В кн.: Проблемы оптической голографии (под ред, Ю. Н. Денисюка). Л., «Наука», 1981, $7-27$.

9. Shkunov, V., Zeldovich, B. Appl. Optics, 18, № 21, 3633-3643 (1979).

10. Каничашвили Ш. Д., Квинихидзе Т. Н. Квант. электроннка, 2, № 7, 1449-1453 (1975), 
11. Romagnoli, M., Levenson, M., Bjorklund, G. J. Opt. Soc. Amer. B, 1, № 4, 571-585 (1984).

12. Саари П. М. В кн.: Тр. Ин-та физики ЭССР, т. 59. Тарту, 1986, 157-184.

13. Каарли P., Сapanyy P. Изв. АН ЭССР. Физ. Матем. (в печати).

14. Апанасевич $\Pi$. А. Основы теории взаимодействия света с веществом. Минск, «Наука и техника», 1977.

15. Келих С. Молекулярная нелинейная оптика. М., «Наука», 1981.

16. Каарли Р. К., Саари П. М., Сынаяле Х. Р. В кн.: Тез. докл.: «Новые методы лазерной спектроскопии молекул в низкотемпературных средах», Таллин, 19-21 мая 1987 г. Таллин, $1987,41-42$.

17. Каарли Р. К., Саари П. М., Сынаялг Х. Р. В кн.: Тез. докл.: «Сверхбыстрые процессы в спектроскопин СПС 87», Вильнюс, 22-25 августа 1987 г. (в печати).
Ннститут физики
Академии наук Эстонской ССР
Поступнла в редакцию
30/VII 1987

R. SARAPUU, P. SAARI

\section{VEKTORVALJA AEGRUUMILINE HOLOGRAAFIA}

Varasemates töödes $\left[{ }^{1,3-5}\right]$ esitatud ultralühikeste valgusimpulsside holograafilise salvestamise ja taastamise protseduuride teooriat on üldistatud keskkondadele, mis lisaks spektraalsele selektiivsusele on tundlikud ka valgusvälja polarisatsiooni suhtes. On näidatud, et sellisel juhul on võimalik kõigi valgussignaalile iseloomulike karakteristikute amplituudi, faasi ja polarisatsiooni - ajalis-ruumilise käitumise salvestamine ja taastamine. On leitud täpsed tingimused, mille puhul signaal taastub täielikult ja ilma moonutuseta.

\section{R. SARAPUU and P. SAARI}

\section{TIME-AND-SPACE DOMAIN HOLOGRAPHY OF VECTOR FIELDS}

Theory of time-and-space domain holography $\left[{ }^{1,3-5}\right]$ have been generalized for the case of variably polarized optical fields. The conditions of distortionless recording and reconstruction of the spatial and temporal behavior of amplitudes, phases and polarization of optical signal have been found. 